\title{
CHARACTERIZING THE GOES-R (GOES-16) GEOSTATIONARY LIGHTNING MAPPER (GLM) ON-ORBIT PERFORMANCE
}

\author{
Scott D. Rudlosky ${ }^{1}$ \\ Steven J. Goodman ${ }^{2}$ \\ William J. Koshak ${ }^{3}$ \\ Richard J. Blakeslee ${ }^{3}$ \\ Dennis E. Buechler ${ }^{4}$ \\ Douglas M. Mach ${ }^{5}$ \\ Monte Bateman ${ }^{5}$ \\ ${ }^{1} \mathrm{NOAA} / \mathrm{NESDIS} / \mathrm{STAR}$ \\ ${ }^{2}$ NOAA/NESDIS/GOES-R Program Office \\ ${ }^{3} \mathrm{NASA} / \mathrm{MSFC}$ \\ ${ }^{4}$ UAHuntsville \\ ${ }^{5}$ USRA
}

\begin{abstract}
Two overlapping efforts help to characterize the GLM performance, the Post Launch Test (PLT) phase to validate the predicted pre-launch instrument performance and the Post Launch Product Test (PLPT) phase to validate the lightning detection product used in forecast and warning decision-making. This paper documents the calibration and validation plans and activities for the first 6 months of GLM on-orbit testing and validation commencing with first light on 4 January 2017. The PLT phase addresses image quality, on-orbit calibration, RTEP threshold tuning, image navigation, noise filtering, and solar intrusion assessment, resulting in a GLM calibration parameter file. The PLPT includes four main activities, the Reference Data Comparisons (RDC), Algorithm Testing (AT), Instrument Navigation and Registration Testing (INRT), and Long Term Baseline Testing (LTBT). Field campaigns are also designed to contribute valuable insights into the GLM performance capabilities. The PLPT tests each contribute to the beta, provisional, and fully validated GLM data.
\end{abstract}

Index Terms - Lightning, GOES-R, GLM, Calibration

\section{INTRODUCTION}

The first Geostationary Lightning Mapper (GLM) sits aboard the first satellite in the Geostationary Operational
Environmental Satellite R-series (GOES-R; which became GOES-16 after reaching geostationary orbit on 29 November 2016). The GLM is the first step in the operational spacebased observing constellation for continuous measurements of total lightning on a global-scale. It builds on a legacy of optical lightning observations from low earth orbit from the NASA Lightning Imaging Sensor (LIS) on the Tropical Rainfall Measuring Mission (TRMM, 1997-2015) and Optical Transient Detector (OTD) on the OrbComm-1 satellite (1995-2000). The GLM on GOES-16 is the first of four instruments that will provide lightning mapping of most of the the western hemisphere through 2036. The initial check-out and post-launch testing is six months at $89.5^{\circ} \mathrm{W}$ longitude followed by a period of extended validation for GOES-16 only of an additional six months. Following the one year of post launch testing the GOES-16 satellite will replace the GOES-E $(75 \mathrm{~W})$ or GOES-W $(137 \mathrm{~W})$ satellites depending on the health of the geostationary satellite constellation at that time.

The ground processing algorithms are an extension of the algorithms developed for the earlier OTD and LIS research instruments [2] (Mach et al. 2007). Concepts for the GLM have been explored since the early 1980s culminating with the single telescope design having high detection efficiency for total lightning with near uniform storm-scale spatial resolution owing to the variable pitch pixel detector array design [1] (Goodman et al. 2013). The high detection efficiency is made possible by the data telemetry bandwidth 
of $7.7 \mathrm{mbps}$. This allows the GLM to be set at more sensitive (lower) detection thresholds allowing up to 100,000 events per second (nominally 40,000 lightning events and the remainder noise) to be transmitted to the ground where the ground processing algorithms filter out the non-lightning events.

The calibration and validation efforts are both critical and challenging because the GLM is the first instrument of this type to operate in geostationary orbit [1]. Pre-launch and onorbit checkout of the instrument performance and algorithms employs a variety of space, airborne, and ground-based instruments. Public, private, and international partnerships provide extensive lightning reference data sets for postlaunch testing and validation. The methodologies, validation tools, and correlative data needed during on-orbit checkout and for continued monitoring have been developed and tested well before launch.

\section{GLM CALIBRATION ACTIVITES}

Two overlapping efforts help to characterize the GLM performance: the vendor-led Post Launch Test (PLT) phase, and the Post Launch Product Test (PLPT) phase led by the GLM science team. A field campaign also contributes to GLM calibration and validation. This paper documents the calibration and validation plans and activities for the first 6 months of GLM operations.

\subsection{Post-Launch Test Phase}

The PLT phase first addresses image quality and on-orbit calibration. GLM periodically collects snapshots of the average background radiance, and the vendor uses a PLT tool to extract and analyze background image metrics. The GLM also allows for on-orbit calibration where a macro in the GLM flight software can dial thresholds for the on-board instrument timing without ground commanding. Setting the Real Time Event Processor (RTEP) detection thresholds is a critical aspect of the PLT. The GLM identifies any positive change in the pixel radiance value that exceeds these programmable detection thresholds, so the RTEP must be tuned carefully. Continuous monitoring of the RTEP thresholds will be required to ensure optimal GLM performance.

Image navigation and noise filtering are also integral to successful GLM operations. User applications require GLM data to be properly navigated to earth-based latitudelongitude coordinates. The Coastline ID is a ground image processing algorithm applied to daytime background images from GLM that identifies coastlines to translate $(x, y)$ to (longitude, latitude) for L1b lightning data. The groundprocessing algorithms (GPAs) include several filters that must be tuned with the GLM collecting data in orbit. The initial PLT tuning is performed on the GLM vendor's GPA implementation. This process is aided by a PLT tool for slicing and displaying event data in time and space. Realtime analysis of the contributions from the various filters along with longer term assessments help to ensure properly tuned filters. The PLT also includes a Solar Intrusion Assessment. During eclipse season, direct solar illumination enters the GOES-16 GLM optics and reaches almost to the focal plane. Approaches for quantifying the degradation (as well as other data quality attributes; e.g. dropped events, possible hot or dead pixels, parallax-caused geolocation errors) and communicating this information to users are being examined.

The output of the PLT is a GLM calibration parameter file. The PLT could result in algorithm changes or new algorithms. In practice, the PLPT will overlap with the PLT. Some instrument-related PLTs must be completed prior to detection efficiency (DE) and False Alarm Rate (FAR) related PLPT assessments. Flexibility is required so that RTEP thresholds can be tentatively set, the DE/FAR tested using PLPT tools, then re-tuning the RTEP thresholds accordingly. This back-and-forth tuning process is vital for thoroughly optimizing the GLM.

\subsection{Post-Launch Product Test Phase}

The PLPT includes four main activities. The first is the Reference Data Comparisons (RDC) which determines whether the GLM is meeting specifications for detecting lightning flashes (Flash Detection Efficiency, FDE), rejecting false flashes (False Flash Rate, FFR), and locating the lightning in time and space. Reference ground-based lightning observation networks have been characterized and shown useful for GLM calibration and validation. Initial emphasis is placed on the well-characterized regional Lightning Mapping Array (LMA) networks and the National Lightning Detection Network (NLDN) over CONUS.

The second activity is Algorithm Testing (AT) that determines whether the GLM algorithm is correctly filtering, geolocating, and clustering the raw Level 0 (L0) data, which contains both backgrounds and lightning. The GLM clustering algorithms were developed with insights gained 
from the OTD and LIS, and are validated using data from various ground-based reference datasets.

The Instrument Navigation and Registration Testing (INRT) determines whether the GLM navigation is meeting specifications. Ground-based lightning detection networks also provide a baseline for this comparison, but additional meteorological observations are included (e.g., WSR-88D radar). Cases of interest are initially investigated followed by more in depth analysis as more data become available.

The Long Term Baseline Testing (LTBT) focuses on determining the present GLM sensitivity, how it compares to the Lightning Imaging Sensor (LIS) and the Optical Transient Detector (OTD), and how it varies over time. The LTBT employs many sensors and observes patterns over various regions and seasons. Field campaigns are also designed to contribute valuable insights into the GLM performance capabilities.

The PLPT tests each contribute to all three data levels, including (1) Beta - minimally validated, may still contain significant errors, (2) Provisional - analyses are sufficient for qualitative and limited quantitative determination of product fitness-for-purpose, and (3) Fully Validated - analyses are sufficient for full qualitative and quantitative determination of product fitness-for-purpose. With more data, better statistics, and more locations come more confidence in the GLM lightning product.

\subsection{GOES-R Field Campaign}

The GOES-R field campaign supports post-launch product validation. A $\sim 6$ week field campaign ( 100 flight hours) has been planned with the high-altitude NASA ER-2 platform coordinated with ground-based and near surface observations (March - May 2017). During Phase 1 (2 weeks) the ER-2 will be based in Palmdale, CA and during Phase 2 (4 weeks) the ER-2 will be based at Warner Robins AFB.

NOAA science participates in all phases of the GOES-R calibration life-cycle. The GOES-R Calibration Working Group (CWG) has provided excellent support in the prelaunch calibration data analysis to verify the pre-launch predicted instrument performance. The CWG has established an in-depth knowledge of the GOES-R instruments through extensive pre- and post-launch support. A strong communication line exists between the CWG and Algorithm Working Group (AWG). Understanding both CWG and
AWG performance concerns and validation limitations fosters the successful cooperation in field campaign activities.

The GLM field campaign focuses on validating the $70 \%$ flash detection efficiency and 5\% false alarm rate. The primary objective is to provide validation of GLM flash detection efficiency day and night over land at well characterized total lightning observation sites (e.g., Northern AL, Lubbock TX, Norman OK, KSC FL, and Wallops/Washington D.C.). A secondary objective is to provide validation of day/night GLM flash detection efficiency at other land locations and over the ocean. A tertiary objective is to provide validation of GLM flash location and time stamp accuracy, as well as GLM image navigation and registration (INR) accuracy.

The GLM field campaign validation priorities include collecting coincident and collocated high altitude data over thunderstorms with the Fly's Eye GLM Simulator (FEGS). The minimum collection set includes over-flights of thunderstorms over total lightning super sites. Emphasis is on large scale convection such as Mesoscale Convective Systems (MCSs) from pre-storm through entire evolution (include all times of day and other storm types). The secondary collection set includes over-flights of thunderstorms at other locations (day/dawn or dusk/night; high/low latitudes; land/ocean; various storm types/regimes). A successful field campaign will include a sample size on the order of 1000 flashes for any region and period of interest in order to characterize detection efficiency, geolocation, and time-stamp accuracy.

\section{SUMMARY}

Is this necessary?

\section{ACKNOWLEDGEMENTS}

Funding for this project was provided by NOAA/NESDIS to the NOAA Cooperative Institute for Climate and Satellites Maryland (CICS-MD) and to the NASA George C. Marshall Space Flight Center. The contents of this paper are solely the opinions of the authors and do not constitute a statement of policy, decision, or position on behalf of NOAA, NASA or the U.S. Government.

\section{REFERENCES}


[1] Goodman S. J. et al., 2013: The GOES-R Geostationary Lightning Mapper (GLM). Atmos. Res., 125-126, 34-49. doi:10.1016/j.atmosres.2013.01.006.

[2] Mach, D. M., H. J. Christian, R. J. Blakeslee, D. J. Boccipio, S. J. Goodman, and W. L. Boeck, 2007: Performance assessment of the Optical Transient Detector and Lightning Imaging Sensor, J. Geophys. Res., 112, D09210, doi:10.1029/2006JD007787. 